\title{
On the staircases of Gyárfás
}

\author{
János Csányi \\ Bolyai Institute, University of Szeged, Hungary \\ csanyi1990@gmail.com \\ Péter Hajnal
}

Bolyai Institute, University of Szeged, Hungary, and

Alfréd Rényi Institute of Mathematics, Hungarian Academy of Sciences, Hungary

hajnal@math.u-szeged.hu

Gábor V. Nagy

Bolyai Institute, University of Szeged, Hungary

ngaba@math.u-szeged.hu

Submitted: Nov 12, 2015; Accepted: Apr 7, 2016; Published: Apr 15, 2016

Mathematics Subject Classification: 05D10

\begin{abstract}
In a 2011 paper, Gyárfás investigated a geometric Ramsey problem on convex, separated, balanced, geometric $K_{n, n}$. This led to appealing extremal problem on square 0-1 matrices. Gyárfás conjectured that any 0-1 matrix of size $n \times n$ has a staircase of size $n-1$.

We introduce the non-symmetric version of Gyárfás' problem. We give upper bounds and in certain range matching lower bound on the corresponding extremal function. In the square/balanced case we improve the $(4 / 5+\epsilon) n$ lower bound of Cai, Gyárfás et al. to $5 n / 6-7 / 12$. We settle the problem when instead of considering maximum staircases we deal with the sum of the size of the longest 0 - and 1staircases.
\end{abstract}

\section{Introduction}

It is well-known (an early remark of Erdős and Rado, nowadays a standard introductory exercise in graph theory courses) that in any red/blue edge coloring of the complete graph a monochromatic spanning tree is guaranteed. It was in 1998 when Károlyi, Pach and Tóth [4] proved the geometric version of this fact: We take $n$ independent points on the plane, connect all the pairs with a line segment, and color them arbitrarily with red and blue colors. Then a non-crossing monochromatic spanning tree is guaranteed. 
The bipartite case of the original graph theoretical question is easy: If we red/blue color the edges of $K_{n, n}$ (the balanced complete bipartite graph) then the largest monochromatic tree has at least $n$ vertices if $n$ is even and $n+1$ if $n$ is odd. Furthermore this bound is optimal, certain coloring doesn't contain a larger monochromatic tree.

The beautiful theorem from [4] led Gyárfás [3] to consider the geometric problem when the underlying graph is a complete bipartite graph: Take any $2 n$ points in convex position on the plane. Assume that a line separates them into two groups of $n$ points. Connect all crossing pairs with a line segment and color them arbitrarily with red/blue colors. What is the size (i.e. the number of vertices) of the largest non-crossing monochromatic tree that you can guarantee? It is conjectured in [2] that the answer is $n$, if $n>1$.

The above/graph version of the basic question can be easily transformed to a matrix version as it was done in [2].

Let $M$ be a $0-1$ matrix. A 0 -staircase is a sequence $\left\{s_{i}\right\}_{i=1}^{\ell}$ of zeroes in $M$ such that $s_{i+1}$ is either to the right of $s_{i}$ in the same row, or it is below $s_{i}$ in the same column $(i=1,2, \ldots, \ell-1)$. (We emphasize that $s_{i}$ and $s_{i+1}$ do not have to be neighbouring elements in $M$.) A 1-staircase is defined similarly on ones of $M . M$ is a homogeneous staircase in $M$ iff it is either a 0 - or a 1-staircase. The length of a homogeneous staircase $S$ is the number of elements in it, we denote it as $|S|$. $S$ can be viewed as $|S|-1$ steps, where the steps can be right steps or down steps, formed by the consecutive elements of $S$. An element of $S$ is a turning point, if it is involved in two steps with different directions. $\operatorname{turn}(S)$ denotes the number of turns in $S$. For example $\operatorname{turn}(S)=0$ iff $S$ includes identical elements from a row, or from a column. $\operatorname{turn}(S)=1$ iff the steps of $S$ are some horizontal (staying in the same row) steps followed by some vertical steps or vice versa. In the first case we say that $S$ is a 7 -staircase. The first elements forming the horizontal steps will be referred as the horizontal hand of our 7 -staircase. The last block of elements that form the vertical steps will be referred as the vertical hand of our 7 -staircase. In the second case we say that $S$ is a $L$-staircase. We can use the notation of horizontal/vertical hands in this case too.

It turns out that the above-mentioned conjecture is equivalent to the following:

Conjecture 1 (Gyárfás' Conjecture). Any 0-1 matrix of size $n \times n$ contains a homogeneous staircase of size $n-1$.

Let $s t(M)$ be the maximum among the lengths of the homogeneous staircases of $M$. Let $s t_{0}(M)$ (resp. $s t_{1}(M)$ ) be the maximum among the lengths of the homogeneous 0 -staircases (resp. 1-staircases) of $M$. Hence $s t(M)=\max \left\{s t_{0}(M), s t_{1}(M)\right\}$.

We define a symmetric and an asymmetric version of the original Ramsey-type question.

$$
\begin{gathered}
s t(n)=\min \left\{\operatorname{st}(M): M \in\{0,1\}^{n \times n}\right\}, \\
\operatorname{st}(n, N)=\min \left\{\operatorname{st}(M): M \in\{0,1\}^{n \times N}\right\} .
\end{gathered}
$$

In the asymmetric case it is obvious that $\operatorname{st}(n, N)=\operatorname{st}(N, n)$. We always assume that $N \geq n$. 
The Gyárfás' conjecture states that $s t(n) \geq n-1$. Since [2] contains an easy example that shows $s t(n) \leq n-1$ (assuming $n>1$ ) we can state Gyárfás' conjecture as $s t(n)=n-1$ if $n>1$.

Instead of $s t(M)=\max \left\{s t_{0}(M), s t_{1}(M)\right\}$ one can investigate

$$
s t_{\Sigma}(M)=s t_{0}(M)+s t_{1}(M) .
$$

It is natural to introduce

$$
\begin{gathered}
s t_{\Sigma}(n)=\min \left\{s t_{\Sigma}(M): M \in\{0,1\}^{n \times n}\right\}, \\
s t_{\Sigma}(n, N)=\min \left\{s t_{\Sigma}(M): M \in\{0,1\}^{n \times N}\right\} .
\end{gathered}
$$

Cai, Grindstaff, Gyárfás and Shull stated a conjecture concerning the function $s t_{\Sigma}(n)$ (see [1]), that turned out to be false (see the final remark in [2], the journal version of [1]). In section 2 we determine the exact value of $s t_{\Sigma}(n, N)$. In section 3 we deal with $s t(n, N)$. We present two constructions (hence we give upper bounds on $\operatorname{st}(n, N))$. We conjecture that our matrices gives the right value of $\operatorname{st}(n, N)$. Our conjecture is consistent with Gyárfás' conjecture. We will be able to prove our conjecture in certain range. Unfortunately the case of square and "near-square" matrices is still unsolved.

In the final section we give a significant improvement of the $\frac{4}{5} n$ bound in [3] (as opposed to the one in [2]). We are going to prove the following theorem.

Theorem 2. For any $M \in\{0,1\}^{n \times n}$

$$
\operatorname{st}(M) \geq \frac{5}{6} n-\frac{7}{12}
$$

\section{The exact value of $s t_{\Sigma}(n, N)$}

We start with an easy construction.

Construction 3. Let $P^{(n, N)} \in\{0,1\}^{n \times N}$ be the matrix, where we have $P_{i, j}^{(n, N)}=0$ iff $i+j \leq\left\lfloor\frac{n}{2}\right\rfloor+1$ or $i+j \geq\left\lfloor\frac{n}{2}\right\rfloor+N+1$.

The following two examples help to understand the formalism:

$$
P^{(6,8)}=\left(\begin{array}{llllllll}
0 & 0 & 0 & 1 & 1 & 1 & 1 & 1 \\
0 & 0 & 1 & 1 & 1 & 1 & 1 & 1 \\
0 & 1 & 1 & 1 & 1 & 1 & 1 & 1 \\
1 & 1 & 1 & 1 & 1 & 1 & 1 & 0 \\
1 & 1 & 1 & 1 & 1 & 1 & 0 & 0 \\
1 & 1 & 1 & 1 & 1 & 0 & 0 & 0
\end{array}\right), \quad P^{(7,8)}=\left(\begin{array}{llllllll}
0 & 0 & 0 & 1 & 1 & 1 & 1 & 1 \\
0 & 0 & 1 & 1 & 1 & 1 & 1 & 1 \\
0 & 1 & 1 & 1 & 1 & 1 & 1 & 1 \\
1 & 1 & 1 & 1 & 1 & 1 & 1 & 0 \\
1 & 1 & 1 & 1 & 1 & 1 & 0 & 0 \\
1 & 1 & 1 & 1 & 1 & 0 & 0 & 0 \\
1 & 1 & 1 & 1 & 0 & 0 & 0 & 0
\end{array}\right)
$$

Note that the 0's form two triangular regions in our matrix (in the upper left and the lower right corners). Because of our convention $(n \leq N)$ there is no 0 -staircase that contains 0 's from both triangular regions. Hence the following fact is easy: 
Observation 4. For any $n \leq N$,

$$
s t_{0}\left(P^{(n, N)}\right)=\left\lceil\frac{n}{2}\right\rceil, \quad s t_{1}\left(P^{(n, N)}\right)=N-1,
$$

and so

$$
s t_{\Sigma}\left(P^{(n, N)}\right)=\left\lceil\frac{n}{2}\right\rceil+N-1
$$

We are going to prove a matching lower bound on $s_{\Sigma}(M)$ for any $M \in\{0,1\}^{n \times N}$.

Theorem 5. For any $n \leq N$,

$$
s t_{\Sigma}(n, N)=\left\lceil\frac{n}{2}\right\rceil+N-1 .
$$

Proof. Let $M \in\{0,1\}^{n \times N}$ arbitrary. Take the elements of the first column, that give the majority of this column. We can assume that they have the common value 1 . Let $a$ be the position of the lowest 1 in the first column. The number of 1's at and above $a$ is at least $\left\lceil\frac{n}{2}\right\rceil$. Let $S_{1}$ the L-staircase centered at $a$. Let $S_{2}$ the staircase formed by the 0's in the row of $a$. It is easy to see that

$$
\left|S_{1}\right|+\left|S_{2}\right| \geq\left\lceil\frac{n}{2}\right\rceil+N-1
$$

This observation proves the theorem.

\section{An upper bound for asymmetric matrices}

In this section we give an upper bound on $\operatorname{st}(n, N)$. We will easily see that our bound is the truth for "wide" enough matrices. (The sharpness of the bound has been validated by computer for a few additional dimensions, too, but only for small $n$ and $N$ values.) We exhibit two constructions. They correspond to two different ranges of shapes.

Construction 6. For $N \geq\left\lfloor\frac{5}{2} n\right\rfloor-1$, we define the matrix $Q^{(n, N)}$ as follows: Let $Q_{i, j}^{(n, N)}=1$ iff one of the following three possibilities holds:

(1) $i \leq\left\lceil\frac{n}{2}\right\rceil$ and $i+j \leq\left\lceil\frac{n}{2}\right\rceil+1$,

(2) $i \leq\left\lceil\frac{n}{2}\right\rceil$ and $\left\lceil\frac{n}{2}\right\rceil+\left\lfloor\frac{\lceil n / 2\rceil+N-1}{2}\right\rfloor+1<i+j$,

(3) $i>\left\lceil\frac{n}{2}\right\rceil$ and $\left\lceil\frac{n}{2}\right\rceil+N-\left\lfloor\frac{\lceil n / 2\rceil+N-1}{2}\right\rfloor<i+j \leq\left\lceil\frac{n}{2}\right\rceil+N$.

The reader is advised to check the example below to understand the structure of matrix $Q^{(n, N)}$ : The first $[n / 2\rceil$ rows of $Q^{(n, N)}$ forms an "isosceles right triangle" of 1 's in the upper left corner (the length of the legs is $\lceil n / 2\rceil$ ), followed by a "parallelogram" of 0 's with horizontal side of length $\left\lfloor\frac{\lceil n / 2\rceil+N-1}{2}\right\rfloor$, and the rest of this upper region is filled with 
1 's. The last $\lfloor n / 2\rfloor$ rows are defined analogously: the triangle in the lower right corner has leg length $\lfloor n / 2\rfloor$, and the parallelogram of 1's has the same width as the parallelogram of 0 's above.

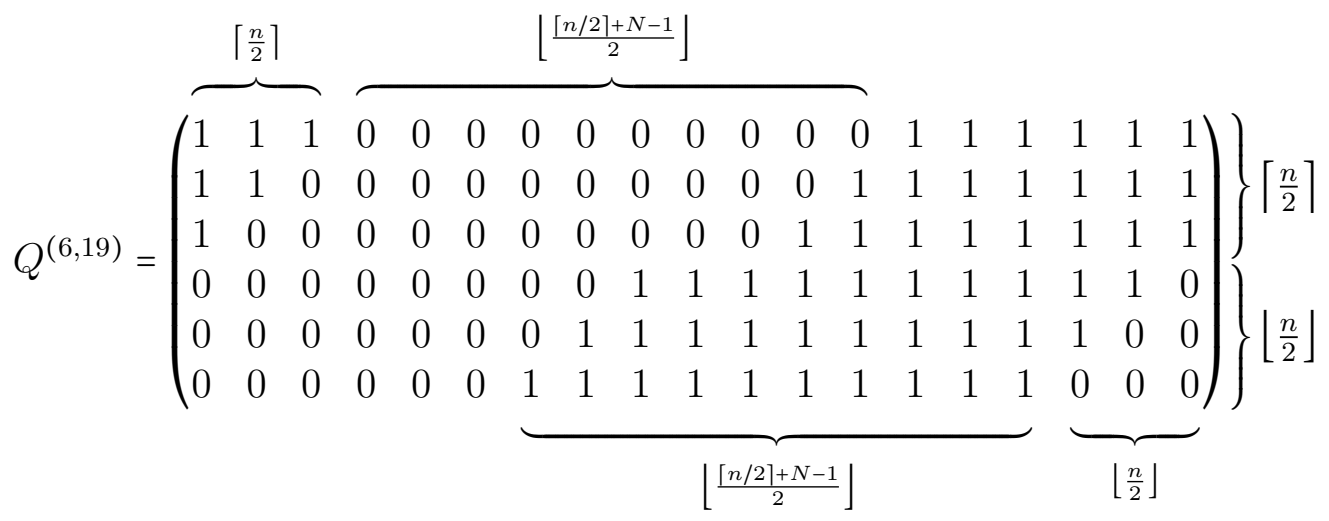

Claim 7. For all $N \geq\left\lfloor\frac{5}{2} n\right\rfloor-1$,

$$
\operatorname{st}\left(Q^{(n, N)}\right)=\left\lceil\frac{\lceil n / 2\rceil+N-1}{2}\right\rceil .
$$

Proof. The 1's in the first row and last column form a 1-staircase of length $\left\lceil\frac{[n / 2\rceil+N-1}{2}\right\rceil$. We show that there are no longer homogeneous staircases in $Q^{(n, N)}$. We prove this for 1-staircases only, the case of 0-staircases is analogous.

The $\lceil n / 2\rceil$-th column, i.e. the column of the rightmost 1 in the upper left corner, precedes the column of the first 1 of the last row. (This can be seen by verifying that

$$
\left\lceil\frac{n}{2}\right\rceil+\left\lfloor\frac{\lceil n / 2\rceil+N-1}{2}\right\rfloor+\left\lfloor\frac{n}{2}\right\rfloor \leq N
$$

holds for $N \geq\left\lfloor\frac{5}{2} n\right\rfloor-1$.) This means that every 1-staircase that starts from the upper left triangle, can only leave this triangle with a right step; which implies that if we translate this triangle of 1's to the right without overlapping any other 1's, the maximal length of 1 -staircases does not decrease. We can translate this triangle to the right by $\left\lfloor\frac{[n / 2\rceil+N-1}{2}\right\rfloor$ positions (the width of the upper parallelogram of 0 's) without overlapping. It is easy to check that in the obtained matrix $\widetilde{Q}$, all the 1 's lies in the region

$$
\left\lceil\frac{n}{2}\right\rceil+N-\left\lceil\frac{\lceil n / 2\rceil+N-1}{2}\right\rceil+1 \leq i+j \leq\left\lceil\frac{n}{2}\right\rceil+N .
$$

Since in a staircase each step increases $i+j$, the sum of the "coordinates" of the actual position, it is obvious that there is no 1 -staircase in $\widetilde{Q}$ with length greater than $\left\lceil\frac{[n / 2]+N-1}{2}\right]$, as required.

Theorem 5 says that $s t_{0}(M)+s t_{1}(M) \geq\left\lceil\frac{n}{2}\right\rceil+N-1$, and so $s t(M) \geq\left\lceil\frac{\lceil n / 2\rceil+N-1}{2}\right\rceil$ holds for every $M \in\{0,1\}^{n \times N}$. Hence we always have $\operatorname{st}(n, N) \geq\left\lceil\frac{[n / 2\rceil+N-1}{2}\right\rceil$. Claim 7 says that $\operatorname{st}(n, N) \leq\left\lceil\frac{\lceil n / 2\rceil+N-1}{2}\right\rceil$, for all $N \geq\left\lfloor\frac{5}{2} n\right\rfloor-1$, so we obtained the following: 
Theorem 8. For all $N \geq\left\lfloor\frac{5}{2} n\right\rfloor-1$,

$$
\operatorname{st}(n, N)=\left\lceil\frac{\lceil n / 2\rceil+N-1}{2}\right\rceil .
$$

Construction 9. For $n<N<\left\lfloor\frac{5}{2} n\right\rfloor-1$, we define the matrix $R^{(n, N)}$ as follows: Let $R_{i, j}^{(n, N)}=1$ iff one of the following three possibilities holds:

(1) $i \leq\left\lceil\frac{n}{2}\right\rceil$ and $i+j \leq\left\lfloor\frac{N-n+2}{3}\right\rfloor+1$,

(2) $i \leq\left\lceil\frac{n}{2}\right\rceil$ and $\left\lfloor\frac{N-n+2}{3}\right\rfloor+\left\lceil\frac{2 n+N-2}{3}\right\rceil+1<i+j$,

(3) $i>\left\lceil\frac{n}{2}\right\rceil$ and $n+\left\lfloor\frac{N-n+2}{3}\right\rfloor<i+j \leq n+N-\left\lceil\frac{N-n-1}{3}\right\rceil$.

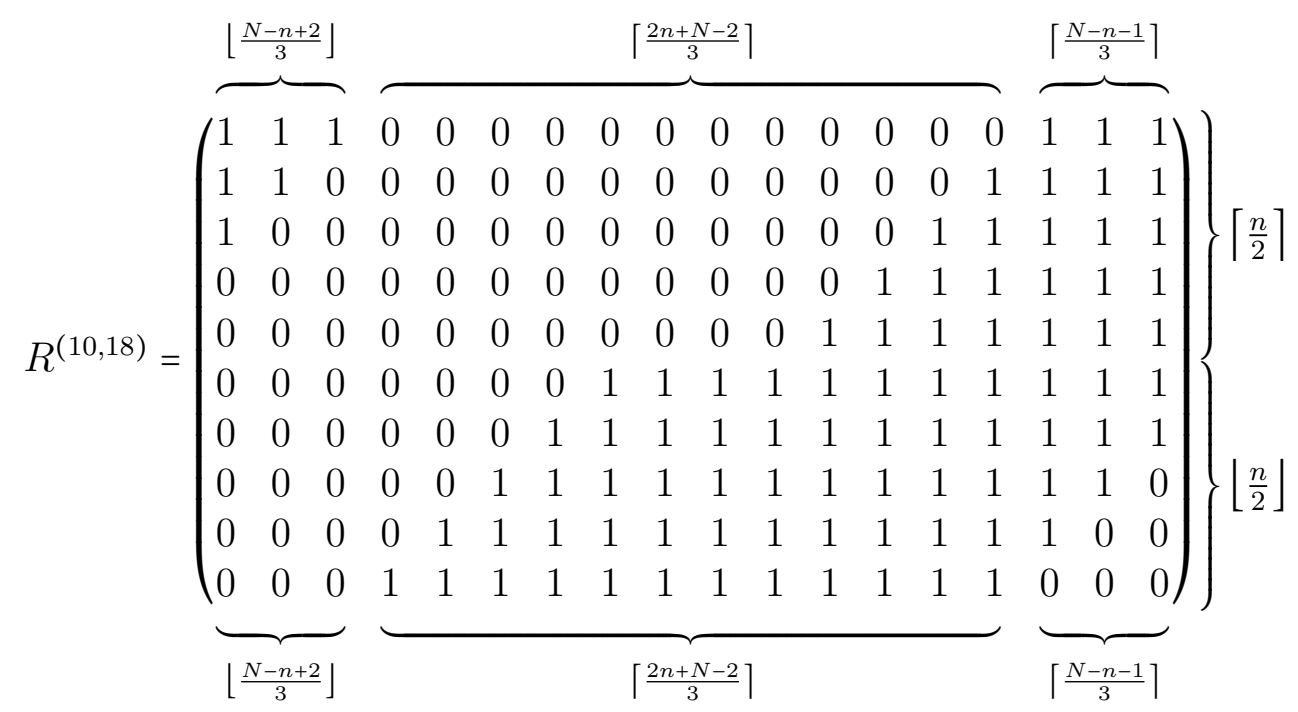

The reader should check that $\left\lfloor\frac{N-n+2}{3}\right\rfloor+\left\lceil\frac{2 n+N-2}{3}\right\rceil+\left\lceil\frac{N-n-1}{3}\right\rceil$ is always equal to $N$, and the conditions imply that $\left\lfloor\frac{N-n+2}{3}\right\rfloor \leq\left\lceil\frac{n}{2}\right\rceil$ and $\left\lceil\frac{N-n-1}{3}\right\rceil \leq\left\lfloor\frac{n}{2}\right\rfloor$, as the above example suggests. After doing this and reading the proof of Claim 7, it should be straightforward to verify the following:

Claim 10. For all $n<N<\left\lfloor\frac{5}{2} n\right\rfloor-1$,

$$
\operatorname{st}\left(R^{(n, N)}\right)=\left\lceil\frac{2 n+N-2}{3}\right\rceil .
$$

Corollary 11. For all $n<N<\left\lfloor\frac{5}{2} n\right\rfloor-1$,

$$
\operatorname{st}(n, N) \leq\left\lceil\frac{2 n+N-2}{3}\right\rceil \text {. }
$$




\section{Improved lower bound, the proof of Theorem 2}

Let $M$ be an arbitrary 0-1 matrix of size $n \times n$. Let $a_{1}$ be the last element of the first row. Without loss of generality we can assume that $a_{1}=1$. Let $a_{2}$ be the last 0 in the first row. Let $a_{3}$ be the top 0 (the 0 with the least first/row index) in the last column. Let $a_{4}$ denote the element/position in the intersection of the column of $a_{2}$ and the row of $a_{3}$.

It is possible that $a_{2}$ is not well defined (the first row does not contain any 0). Since in this case Gyárfás' conjecture is obviously true we assume that $a_{2}$ (and $a_{3}$ as well) is well defined.

$$
\left.\left(\begin{array}{ccccc}
\overbrace{}^{\cdots} & 0_{a_{2}} & 1 & \cdots & 1_{a_{1}} \\
\vdots & & & & 1 \\
& & & \vdots \\
& & & & 1 \\
\vdots & & ?_{a_{4}} & & 0_{a_{3}} \\
& \vdots & & \vdots
\end{array}\right)\right\} w_{1} \text { many 1's 1's }
$$

The summary of our notations, with some references to future parameters.

Observation 12. If $a_{4}=0$ then Gyárfás' conjecture is true for our matrix.

Proof. We consider two staircases: (1) The one that is formed by the 1's in the first row and the 1's in the last column. (2) The one that is formed by the 0's of the first row, $a_{4}$, the 0 's above and on its right side of $a_{4}$, and the 0 's of the last column. The staircase described in (1) is a 1-staircase, and (2) defines a 0-staircase.

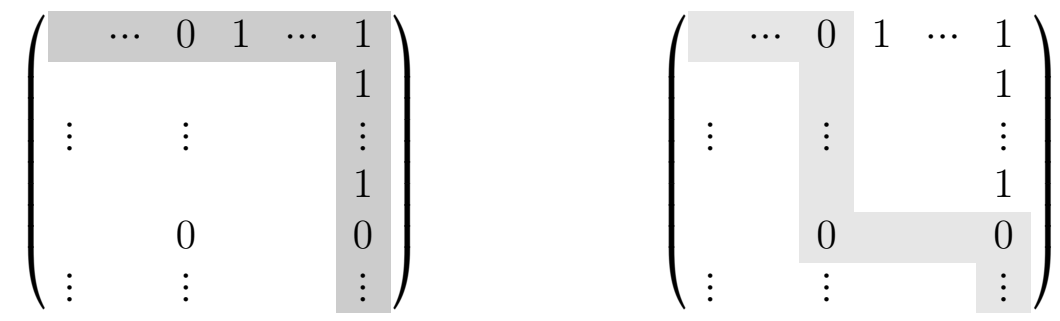

The highlighted elements just show the shape of the staircase. For the staircase we must consider only the elements with the same value as the turning points have.

It is easy to check that the sum of the length of the above two staircases is at least $2 n$. Hence the longer one proves the conjecture.

In the rest of the proof we assume that $a_{4}=1$. Let $S_{i}$ denote the 7 -staircase centered at $a_{i}(i=1,2,3,4)$. Let $s_{i}=\left|S_{i}\right|$. 

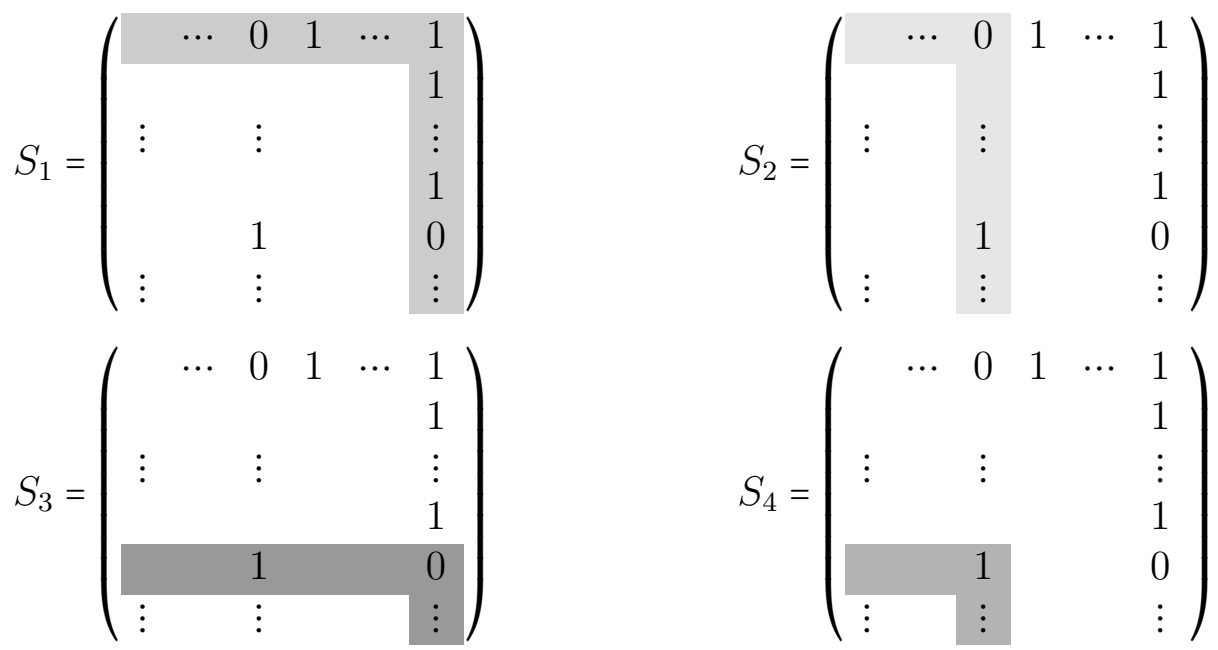

\section{Observation 13.}

$$
2 s_{1}+s_{2}+s_{3}+s_{4}=4 n-3+x_{1}+y_{0}+z_{0}+w_{1} .
$$

where $x_{1}$ denotes the number of 1 's in the first row before $a_{2}, y_{0}$ denotes the number of 0 's in the column of $a_{2}$ between $a_{2}$ and $a_{4}, z_{0}$ denotes the number of 0 's in the row of $a_{3}$ between $a_{4}$ and $a_{3}$, and $w_{1}$ denotes the number of 1 's in the last column under $a_{3}$.

Proof. $s_{1}$, the number of 0's in the horizontal hands of $S_{2}$, and the number of 0's in the vertical hands of $S_{3}$ add up to $2 n-1$.

$s_{4}$, the number the number of 0's before $a_{4}$, and the number of 0's under $a_{4}$ (these 0's are counted in $s_{2}$ and $s_{3}$ ) add up to the number of the shaded positions in the figure of $S_{4}$. The second $s_{1}$ term counts the 1's in last block of 1's of the first row and the 1's in the top block of 1's of the last column. The considered contribution of the sum of length give us $2 n-2$.

It is easy to see that the not counted contribution of the staircases gives us the last four terms on the right hand side.

We can repeat the same argument with exchanging the role of rows and columns. Then the role of $a_{1}$ will be played by the last element of the first column (i.e. the first element of the last row). Let $a$ be the value of this element. $\bar{a}$ denotes $1-a$.

$$
w_{a}^{\prime} \text { many } a \text { 's }\left\{\begin{array}{cccccc}
\vdots & & & \vdots & & \vdots \\
\bar{a}_{a_{3^{\prime}}} & \cdots & & a_{a_{4^{\prime}}} & \cdots & \\
a & & & \vdots & & \vdots \\
\vdots & & & \vdots & \\
a & & & & \\
a_{a_{1^{\prime}}} & \cdots & a & \bar{a}_{a_{2^{\prime}}} & \underbrace{}_{x_{a}^{\prime} \text { many }} a^{\prime} \mathrm{s}
\end{array}\right.
$$


All previous notations can be introduced in this setting (we use the same letters and $\mathrm{a}^{\prime}$ to distinguish from the originals). Our previous observation leads to

$$
2 s_{1}{ }^{\prime}+s_{2}{ }^{\prime}+s_{3}{ }^{\prime}+s_{4}{ }^{\prime}=4 n-3+x_{a}{ }^{\prime}+y_{\bar{a}}{ }^{\prime}+z_{\bar{a}}{ }^{\prime}+w_{a}{ }^{\prime} .
$$

The order of magnitude of Gyárfás' original bound is already proven by our observations. We see some additional terms too, but the usage of them requires a case analysis.

Case 1: $s_{1}+s_{a}^{\prime} \geq 2 n-2$. In this case the longer of $S_{1}$ and $S_{1}{ }^{\prime}$ proves Gyárfás' conjecture and we are done.

Case 2: $s_{1}+s_{a}{ }^{\prime}<2 n-2$.

$S_{1}$ is a 7 -staircase, $s_{1}$ is its size. Let $s_{1}^{-}$denote the size of the horizontal hand of $S_{1}$, and let $s_{1}^{\prime}$ denote the size of its vertical hand. We introduce similar notation for its symmetric pair, the staircase, broken at the left bottom element of $M$ (its size is denoted by $\left.s_{a}{ }^{\prime}\right)$.

Since $s_{1}=s_{1}^{-}+s_{1}^{\prime}-1$ and $s_{a}^{\prime}=s_{a}^{-\prime}+s_{a}^{\prime \prime}-1$, we have

$$
2 n-2>s_{1}+s_{a}^{\prime}=\left(s_{1}^{-}+s_{1}^{\prime}-1\right)+\left(s_{a}^{-\prime}+s_{a}^{\prime \prime}-1\right)=\left(s_{1}^{-}+s_{a}^{-\prime}\right)+\left(s_{1}^{\prime}+s_{a}^{\prime \prime}\right)-2 .
$$

Without loss of generality we can assume, that $s_{1}^{-}+s_{a}^{-\prime}<n$. It is obvious that we are guaranteed to have a column in our matrix that has a 0 in the first/top position, $a_{5}$ and has an $\bar{a}$ at the last/bottom position, $a_{6}$. In the next picture we shaded this column and extended it to a staircase shape with further shaded positions (we are talking about staircase SHAPE, not about staircase!):

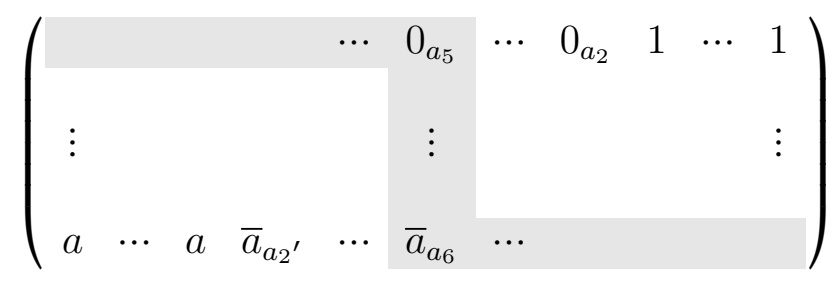

We define two staircases among the shaded elements. We distinguish two subcases:

Subcase 1: $0=\bar{a}$. Let $S_{5}$ be the sequence of shaded 0's and let $S_{6}$ be the 1's of the shaded column.

Subcase 2: $0 \neq \bar{a}$. Let $S_{5}$ be a 7 -staircase centered at $a_{5}$, and Let $S_{6}$ be a L-staircase centered at $a_{6}$.

In both cases $\left|S_{5}\right|+\left|S_{6}\right|$ counts all shaded elements (that is $2 n-1$ elements) except the shaded 1's in the first row and the shaded $a$ 's in the last row. We can upper bound their number with $x_{1}+x_{a}{ }^{\prime}$. We obtained that

$$
\left|S_{5}\right|+\left|S_{6}\right| \geq 2 n-1-x_{1}-x_{a}{ }^{\prime} .
$$

Hence

$$
2 s_{1}+s_{2}+s_{3}+s_{4}+2 s_{1}{ }^{\prime}+s_{2}{ }^{\prime}+s_{3}{ }^{\prime}+s_{4}{ }^{\prime}+\left|S_{5}\right|+\left|S_{6}\right| \geq 10 n-7+y_{0}+z_{0}+w_{1}+y_{\bar{a}}{ }^{\prime}+z_{\bar{a}}{ }^{\prime}+w_{a}{ }^{\prime} .
$$

We investigated 10 staircases. A weighted average of their length is at least $1 / 12(10 n-7)=$ $5 n / 6-7 / 12$. So the longest staircase in our proof proves the theorem. 
Remark 14. Before we started the main streamline of the proof we excluded $a_{4}=0$ (and later we also excluded $\left.a_{4}{ }^{\prime}=\bar{a}\right)$. There, we used staircases $S$ such that $\operatorname{turn}(S)=3$. All the other staircases in our proof have turning points at most 2 . So our theorem can be stated in a stronger form: Any $M \in\{0,1\}^{n \times n}$ contains a staircase that has length at least $5 n / 6-7 / 12$, and it has at most 3 turning points.

Gyárfás exhibited an example that shows that his $4 n / 5$ cannot be improved if we use only staircases with at most 1 turning point.

We do not know any bound for the case when we are allowed to use only staircases with at most 2 turning points.

\section{Acknowledgement}

The second author was partially supported by TÉT_12_MX-1-2013-0006.

\section{References}

[1] S. Cai, G. Grindstaff, A. Gyárfás, and W. Shull, Noncrossing Monochromatic Subtrees and Staircases in 0-1 Matrices, http://www.bsmath.hu/staircasefinal.pdf under the homepage of Budapest Semester. A preliminary version of [2].

[2] S. Cai, G. Grindstaff, A. Gyárfás, and W. Shull, Noncrossing Monochromatic Subtrees and Staircases in 0-1 Matrices, Journal of Discrete Mathematics Volume 2014, Article ID 731519, 5 pages, Hindawi Publishing Corporation, doi:10.1155/2014/731519.

[3] A. Gyárfás, Ramsey and Turán-type problems for non-crossing subgraphs of bipartite geometric graphs, Annales Univ. Sci. Budapest, 54 (2011), 47-56.

[4] G. Károlyi, J. Pach, G. Tóth, Ramsey-type results for geometric graphs, I., Discrete and Computational Geometry, 18 (1997), 247-255.

[5] G. Károlyi, J. Pach, G. Tóth, P. Valtr, Ramsey-type results for geometric graphs, II., Discrete and Computational Geometry, 20 (1998), 375-388. 